\title{
Comment on "Energetic particle sounding of the magnetospheric cusp with ISEE-1” by K. E. Whitaker et al., Ann. Geophys., 25, 1175-1182, 2007
}

\author{
S. A. Fuselier, S. M. Petrinec, and K. J. Trattner \\ Lockheed Martin Advanced Technology Center, Palo Alto, CA, USA \\ Received: 30 August 2008 - Revised: 30 November 2008 - Accepted: 10 December 2008 - Published: 26 January 2009
}

\section{Introduction}

In a recent paper, Whitaker et al. (2007) (hereafter referred to as paper 1) described energetic particle observations in the magnetospheric cusp from the International Sun Earth Explorer (ISEE)-1 spacecraft. This event, on 30 October 1978, has been studied by the authors in a previous publication (Whitaker et al., 2006) and some ISEE-2 observations from this event were presented by Phillips et al. (1993). In paper 1, the authors argue that energetic particle pitch angle distributions observed in and "above" the cusp demonstrate that 24$44.5 \mathrm{keV}$ ions observed in the region cannot be from the bow shock. In particular, they present energetic ion fluxes at several pitch angles that appear to indicate that the $24-44.5 \mathrm{keV}$ ions propagate to the spacecraft from "below" (presumably from the cusp). From these observations, they conclude that: "if the bow shock was the source, a large population of particles would be observed from above", "The deficit in particles with pitch angles less than about $60^{\circ}$ clearly shows that the bow shock is not providing the main source of the CEPs [Cusp Energetic Particles] ... The particles originate locally indicating that the source of CEPs could be from the dayside [magnetosphere] or even within the cusp." "The [energetic] particles streaming from below are therefore lost on these [cusp] open field lines and must be generated locally to have flux enhancements that persist for more than two hours", and finally, "The observation of energetic particles after the spacecraft had exited the [Cusp Diamagnetic Cavity] and was above the cavity supports the theory of local energization."

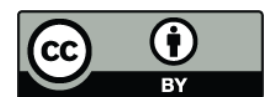

Correspondence to: S. A. Fuselier (stephen.a.fuselier@lmco.com)
These results were obtained by studying pitch angle distributions of ions that represent a small fraction of the total ion density or energy. In this comment, the bulk of the plasma is investigated and it is concluded that the claims made in paper 1 are not substantiated. In particular, the $24-44.5 \mathrm{keV}$ ions in paper 1 are simply part of a much denser thermal ion population whose flow characteristics are dictated by the reconnection geometry and not by a local acceleration source. Thus, unless the local acceleration mechanism is extended to the entire ion population, the use of the pitch angle information in paper 1 is not justified and an alternative explanation for the origin of the $24-44.5 \mathrm{keV}$ ions is needed.

The entire ion population appears to have been accelerated at a low-latitude reconnection site and is expanding into the lower magnetic field region that is poleward and tailward of this site. Using a technique employed by Fuselier et al. (2002) and more recently by Trattner et al. (2009) for energetic particle events, the reconnected field lines are traced back to the solar wind. This tracing demonstrates that they originated in the quasi-parallel region of the Earth's bow shock (prior to reconnection) and, contrary to the conclusion in paper 1, the bow shock is a strong candidate for the 24$44.5 \mathrm{keV}$ ions observed continuously in this event.

\section{ISEE-1 and -2 locations}

Figure 1 shows a schematic of the magnetic field configuration encountered by the ISEE- 1 and -2 spacecraft on their outbound trajectory through the magnetosphere on 30 October 1978. The schematic is drawn in the noon-midnight plane although the spacecraft were located on the dawnside near 09:00 LT. The two ISEE spacecraft are separated by 1.3 Earth Radii $\left(R_{E}\right)$, with ISEE-2 somewhat above (by $0.6 R_{E}$ ) and mostly sunward (by $1 R_{E}$ ) of ISEE-1. The period of

Published by Copernicus Publications on behalf of the European Geosciences Union. 


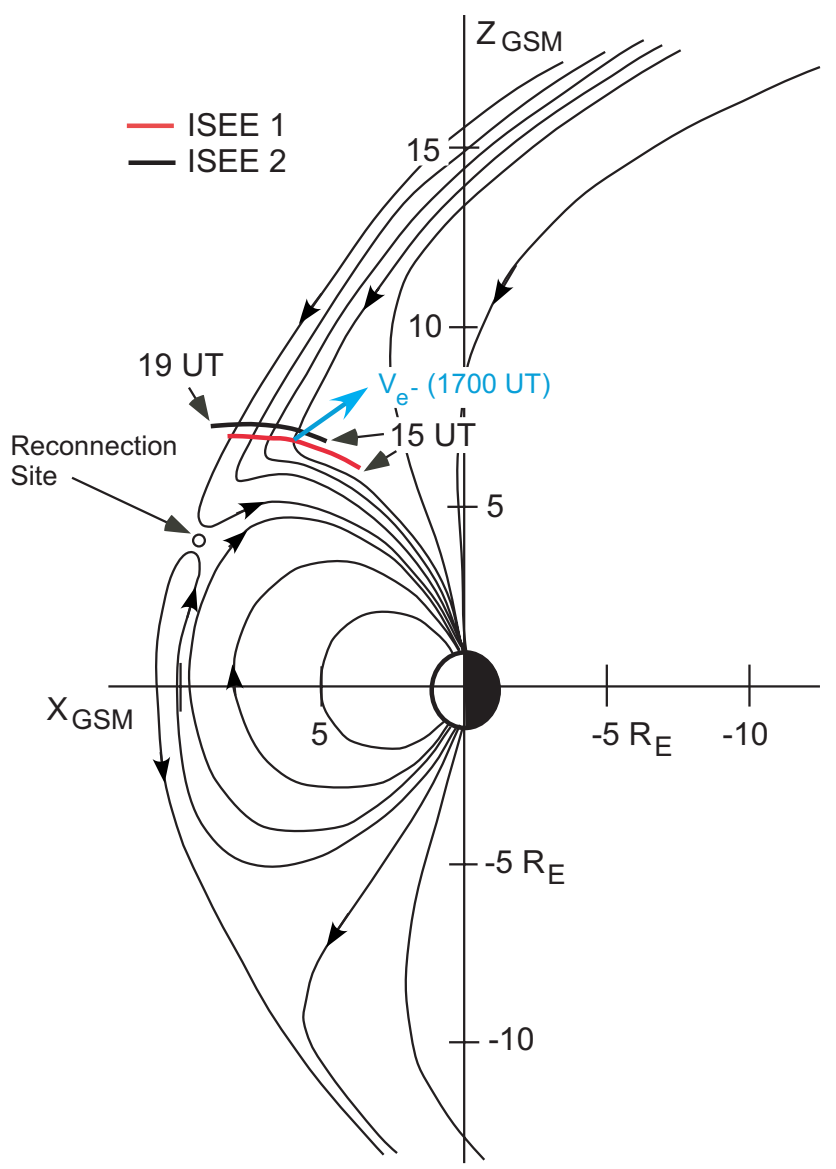

Fig. 1. Schematic of the magnetospheric magnetic field configuration encountered by the ISEE spacecraft from 15:00-19:00 UT. A low latitude reconnection site connects magnetic field lines from the northern magnetosheath with previously closed field lines in the magnetosphere. The spacecraft encounter these open field lines as they convect poleward and tailward from the reconnection site.

interest (from paper 1) is from 15:00 UT to 19:00 UT. During that period, ISEE-1 remained in the magnetosphere. However, Phillips et al. (1993) showed that ISEE-2 was in the high-latitude magnetosheath from about 16:05 to 16:50 UT with one brief return to the magnetosphere/boundary layer at about 16:45 UT. Thus, both spacecraft were relatively close to the magnetopause (i.e. within about $1 R_{E}$ ) for most of the interval of interest.

The schematic magnetic field configuration in Fig. 1 was obtained as follows. The Tsyganenko 96 magnetic field model was used to determine the basic structure of the inner magnetosphere. A reconnection site was located at low latitudes, based on the southward IMF orientation during the event and the interpretation of the event by Phillips et al. (1993). The magnetic field lines poleward of this reconnection site were drawn to match the magnetic field configuration shown in paper 1 (paper 1, Fig. 1). In particular, for the interval of interest, the $B_{x}$ GSM component was positive or zero, the $B_{z}$ GSM component was mainly negative and, in general $\left|B_{x}\right|<\left|B_{z}\right|$. The $B_{y}$ GSM component (not shown in Fig. 1) was positive, consistent with the spacecraft location at high latitude, poleward of the cusp, and on the dawnside of the magnetosphere.

In Fig. 1, the magnetic field orientation dictates the location of the spacecraft. Namely, because the $B_{x}$ GSM component is positive and the $B_{z}$ GSM is negative, the spacecraft must be located poleward of the cusp. If they were located equatorward of the cusp, then the $B_{x}$ component would be negative, the $B_{z}$ component would be either slightly positive or slightly negative, and, in general, $\left|B_{x}\right|$ would be greater than $\left|B_{z}\right|$. The magnetic field orientation indicates that the spacecraft is poleward and tailward of the low-latitude reconnection site and is also poleward of the cusp (this latter interpretation is in agreement with the one in paper 1). There are occasions when the $B_{z}$ component is positive. During these periods (e.g. near 18:00 UT in paper 1, Fig. 1), the $B_{x}$ component is nearly zero. Presumably, during these brief periods, the ISEE-1 spacecraft is closer to the reconnection site and closer to the "kink" in the field line that points northward (but, unlike in Fig. 1, has no $B_{x}$ component). These field lines are still located poleward of the cusp and, at those brief periods, the spacecraft must be very close to the magnetopause. A second explanation for these brief periods is that the reconnection site has moved poleward of the spacecraft. In this interpretation, the spacecraft is very near the reconnection site as well as the magnetopause. Given the available information, it is not possible to determine the distance to the reconnection site. However, the IMF orientation and the reconnection model suggest that the reconnection site was several Earth Radii equatorward of the spacecraft. The IMF remained southward for the entire interval of interest. There was a change in the IMF $B_{y}$ component from negative to positive at about 16:30 UT, which moved the model reconnection site further equatorward. Thus, the magnetic field configuration in Fig. 1 is probably representative of the entire interval of interest.

Identifying the magnetopause location poleward of the cusp is somewhat difficult because there is little difference between the magnetic field orientation inside and outside the high latitude magnetopause as the reconnected field line loses its "kink" at low latitudes. However, from the combined ISEE-1 (paper 1) and ISEE-2 (Phillips et al., 1993) observations, the magnetopause must be less than $\sim 1 R_{E}$ sunward of the blue velocity vector that is labelled 17:00 UT in Fig. 1. ISEE- 2 observed energetic ions $(8<E<30 \mathrm{keV})$ in the magnetosphere and also in the magnetosheath near the magnetopause (Phillips et al., 1993, Plate 1).

ISEE-1 is on open field lines in the magnetosphere. These field lines have one foot in the ionosphere. The other end of the field line (that convected into the magnetosphere from the magnetosheath) is located at high northern latitudes, tailward of the terminator, and on the dawnside of the magnetosphere. These field lines have reconnected at low latitudes and are 


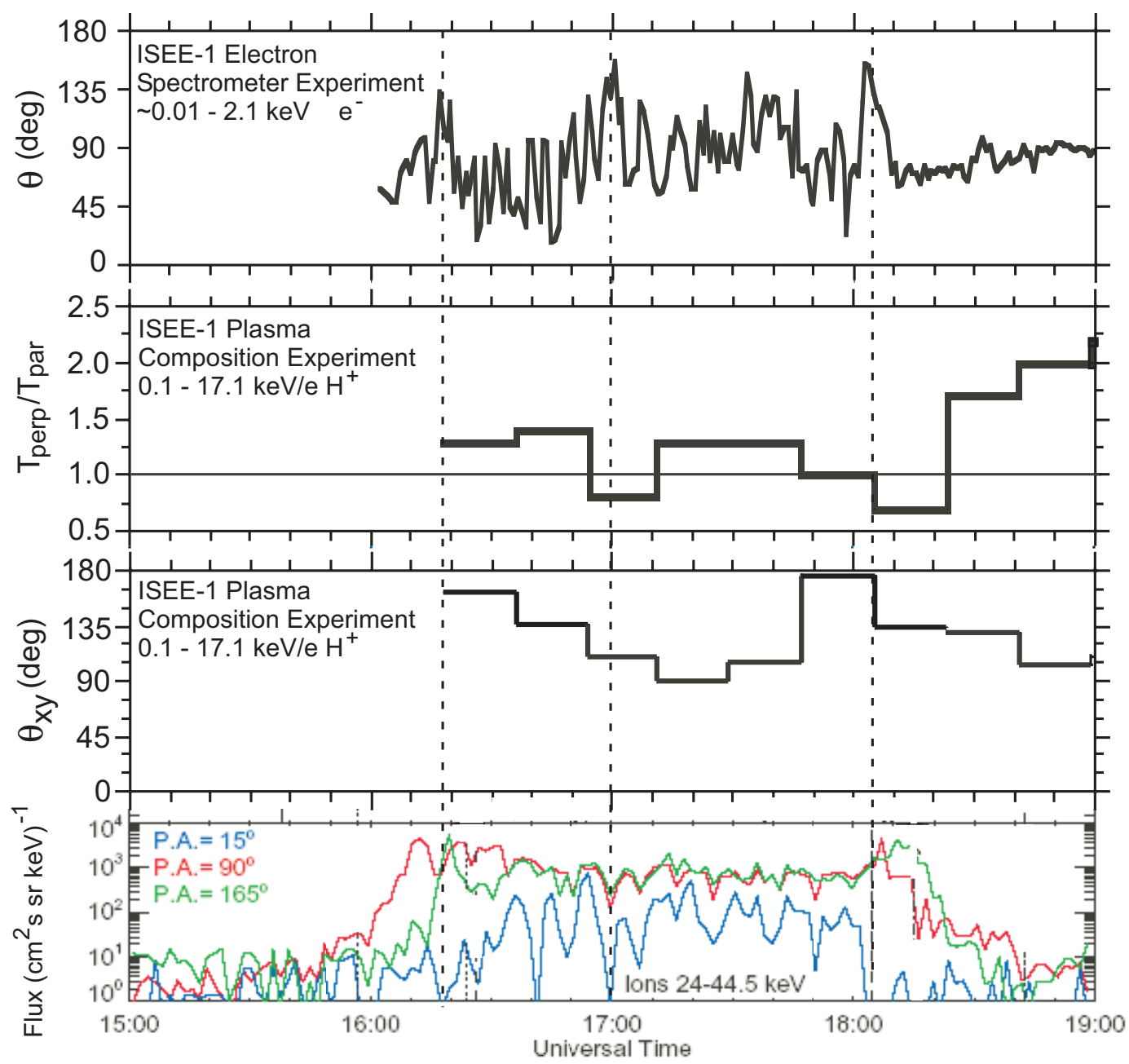

Fig. 2. Top to bottom, Angle between the electron bulk flow direction and the magnetic field, ratio of perpendicular and parallel temperatures for $\mathrm{H}^{+}$(from $0.1-17.1 \mathrm{keV} / \mathrm{e}$ ), angle between the $\mathrm{H}^{+}$flow in the ecliptic plane and the ecliptic plane magnetic field, and energetic (24$44.5 \mathrm{keV}$ ) ion fluxes at three pitch angles. Dashed lines show three times when the energetic ion flux anti-parallel to the magnetic field is much larger than the parallel flux and the corresponding electron flows are directed nearly anti-parallel to the magnetic field. At the exit from the cusp (starting at 17:50 UT), energetic ion fluxes perpendicular to the magnetic field are less than those along the field and the thermal ion population shows an anisotropy $T_{\perp} / T_{\|}<1$. Later, energetic ion fluxes perpendicular to the magnetic field are greater than those along the field and the thermal ion population shows an anisotropy $T_{\perp} / T_{||}>1$. These comparisons demonstrate that the energetic ion population is simply part of an anisotropic thermal population that is convecting poleward and tailward.

expanding poleward and tailward, ultimately becoming lobe field lines in the magnetotail. During this expansion, plasma slows down from its initially accelerated state (at the reconnection site), and, because plasma $\beta \sim 1$, ions and electrons could have a significant "cross-field drift" as they drag the magnetic field line tailward. In models and observations that are typical of low latitude reconnection, plasma on field lines poleward of the cusp either entered the magnetosphere locally (if the magnetopause is very close to the spacecraft) or entered the magnetosphere at low latitudes, propagated along reconnected field lines to the ionosphere, mirrored, and returned to high latitudes.

\section{ISEE-1 plasma observations}

The ISEE-1 spacecraft was equipped with several plasma instruments that measured bulk plasma properties in the thermal energy range (e.g. at energies $<1 \mathrm{keV}$ ). Most of these data are no longer available or easily accessible for study. However, data are still readily available from two plasma instruments on the spacecraft, the Electron Spectrometer Experiment (Oglivie et al., 1978) and the Plasma Composition Experiment (Shelley et al., 1978). The Electron Spectrometer Experiment measured three-dimensional electron distributions in the energy range from $10 \mathrm{eV}-2 \mathrm{keV}$ and the Plasma Composition Experiment measured two-dimensional 


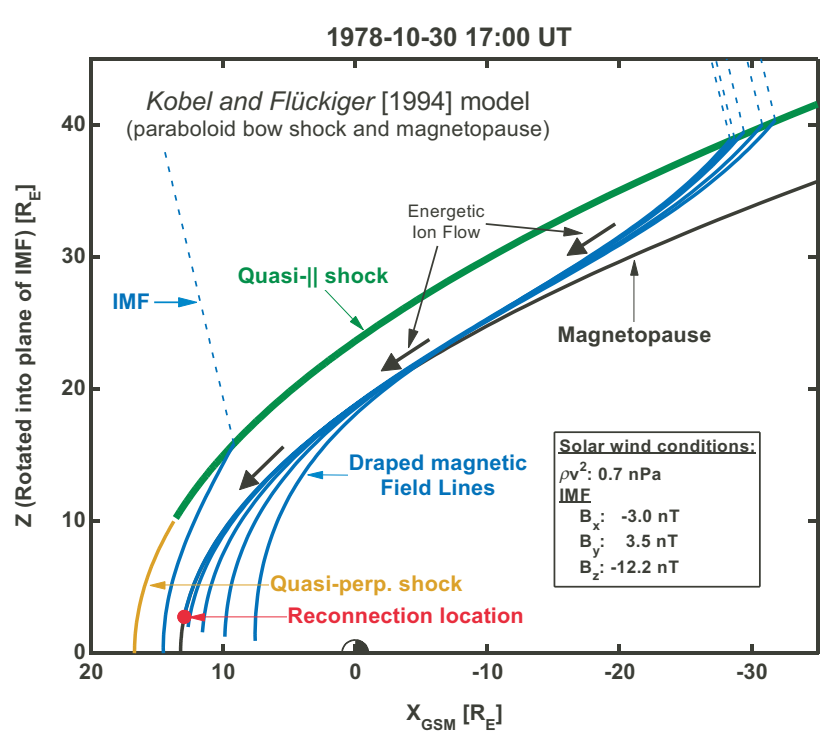

Fig. 3. Schematic illustration of the IMF convecting into the magnetosheath for conditions near 17:00 UT. Field lines convecting through the magnetosheath thread the quasi-parallel bow shock region. In this region, ions are ubiquitously accelerated up to energies of $\sim 150 \mathrm{keV} / \mathrm{e}$. As the field line drapes against the magnetopause, it reconnects at the low latitude reconnection site. Thermal plasma and energetic ions on the field line undergo acceleration at the reconnection site, propagate to the ionosphere, and return to high latitudes. The reconnected field line convects to high latitudes, where the thermal and energetic ion populations were observed by the ISEE-1 spacecraft.

ion distributions (in the ecliptic plane) in the energy range from $\sim 100 \mathrm{eV}$ to $17.1 \mathrm{keV}$. These two plasma instruments have advantages and disadvantages. For electron measurements, the advantages are that 3-D electron distributions in the thermal energy range are measured reasonably rapidly (data used here are averaged to $48 \mathrm{~s}$ time resolution) and the disadvantage is that, for low flow velocities during the event, flow directions are not well determined and fluctuate by large angles. This disadvantage is the direct result of the difficulty in measuring the velocity of a distribution that is moving at $<100 \mathrm{~km} / \mathrm{s}$ but has a thermal speed of $\sim 1000$ $2000 \mathrm{~km} / \mathrm{s}$. For ion composition measurements, the advantages are that individual ion species are measured in the thermal energy range and the disadvantages are that only 2-D (in the ecliptic plane) flow velocities are determined at relatively low ( $\sim 10 \mathrm{~min}$ ) time resolution. Unfortunately, for both the electrons and the ion composition measurements, there is a data gap from 15:00 to about 16:00 UT on 30 October 1978.

Figure 2 compares data from these plasma instruments to data from paper 1 (i.e. from the ISEE-1 Medium Energy Particle Experiment). From top to bottom the panels show time profiles from 15:00 to 19:00 UT of $\vartheta$, the angle between the electron flow velocity and the magnetic field, the perpendicular to parallel ion temperature for the thermal ion distribution, $\vartheta_{x y}$, the angle between the ecliptic plane $\mathrm{H}^{+}$flow velocity and ecliptic plane components of the magnetic field, and the $24-44.5 \mathrm{keV}$ flux at $15^{\circ}, 90^{\circ}$, and $165^{\circ}$ pitch angles (these energetic particle fluxes are from paper 1, Fig. 1).

In paper 1 , it is argued that the energetic ion fluxes (bottom panel of Fig. 2 in this comment) show a deficit of $15^{\circ}$ pitch angle particles, indicating that the energetic particles are coming from "below" (equatorward) of the spacecraft. The top panel and third panel of Fig. 2 show that the bulk of the plasma is behaving in a similar manner as the energetic ions. That is, when the energetic particle fluxes at $15^{\circ}$ pitch angle are several orders of magnitude smaller than the fluxes at $165^{\circ}$ pitch angle flux, the bulk of the plasma is flowing nearly anti-parallel to the magnetic field. Three of these times are shown by dashed lines at 16:18, 17:00, and 18:05 UT in Fig. 2. At these instances, the bulk plasma flow in the ecliptic plane is nearly $180^{\circ}$ and the overall bulk plasma flow (including the $B_{z}$ direction) is $\sim 135^{\circ}$ or greater. Figure 1 (blue vector) shows the bulk plasma flow direction at 17:00 UT. At this time, the X component of the velocity is negative, indicating that the plasma is convecting tailward as well as poleward. At other times, when the energetic particle fluxes at $15^{\circ}$ and $165^{\circ}$ pitch angles are within one order of magnitude of one another, the bulk plasma is flowing across the field $\left(\vartheta \sim 90^{\circ}\right)$, but the flow is still tailward. Other ion species (e.g. solar wind $\mathrm{He}^{++}$and magnetospheric $\mathrm{O}^{+}$(not shown)) exhibit similar flow characteristics.

Like the energetic ions, the bulk plasma is also anisotropic. Furthermore, both populations have similar anisotropies. In particular, the exit from the cusp region (starting at 17:50 UT) shows that, just after 18:00UT, energetic particle fluxes at $165^{\circ}$ are greater than fluxes at $90^{\circ}$ and, for the bulk of the $\mathrm{H}^{+}$ distribution, $T_{\perp} / T_{\|}<1$. Shortly after, at 18:20 UT, the reverse is true, energetic particle fluxes at $165^{\circ}$ are less than fluxes at $90^{\circ}$ and, for the bulk of the $\mathrm{H}^{+}$distribution, $T_{\perp} / T_{\|}>1$. Thus, the characteristics of the bulk of the plasma are reflected in the energetic particle population. The dramatic change in $\mathrm{H}^{+}$anisotropy is associated with a significant change in plasma $\beta$ (not shown). From 16:00-18:00 UT, the plasma density was $\sim 6 \mathrm{~cm}^{-3}$, the temperature was $\sim 1 \mathrm{keV}$, and the magnetic field strength was $\sim 30 \mathrm{nT}$, resulting in a plasma $\beta \sim 0.5-1$. After 18:20 UT, the density decreases dramatically and plasma $\beta$ decreases to $\sim 0.05-0.1$. Because plasma $\beta$ is lower, the $\mathrm{H}^{+}$distribution can be significantly more anisotropic without generating electromagnetic ion cyclotron waves and relaxing the anisotropy.

\section{Origin of $24-44.5 \mathrm{keV}$ ions}

Having established that the energetic ion population investigated in paper 1 is behaving the same way as the bulk of the plasma (and therefore is not accelerated locally in the cusp); the origin of this energetic ion population can be revisited. Figure 3 shows draping of magnetic field lines in 
a bow shock-magnetosheath-magnetopause model. For this draping, the $\mathrm{Z}$ axis is rotated into the plane of the IMF at 17:00 UT. Several field lines are shown, including one (with the "IMF" label) that has passed through the bow shock and is in the process of draping against the magnetopause. Others are shown at a later time when they have draped and are starting to convect around the magnetopause. Based on this draping model and the IMF orientation, the location of the reconnection site is shown by the red dot. Field lines reconnect at that location and form a connection between the solar wind and ionosphere. As the solar wind field line convects into the magnetosheath, it threads the quasi-parallel bow shock (the green region on the shock in Fig. 3), where there is ubiquitous acceleration of solar wind ions to energies $\sim 150 \mathrm{keV}$. These energetic ions propagate back against the flow in the magnetosheath (Fig. 3) to the reconnection site. They are observed by ISEE- 2 in the magnetosheath, adjacent to the magnetopause (Phillips et al., 1993, Plate 1).

Further acceleration of energetic ions and the thermal plasma should occur at the reconnection site. The low and high energy ions propagate to the ionosphere along the newly open field lines, mirror, and return to high latitudes. The reconnected field line continues to convect tailward (Fig. 1) and the entire ion population propagates outward from the cusp with a significant cross field drift. These are the plasma conditions encountered by ISEE-1 on its outbound orbit. Since the general configuration of the magnetic field both inside and outside the magnetopause remains the same throughout the time period of interest, this process of filling the flux tube with energetic ions, reconnecting it, and then convecting the reconnected field poleward and tailward from the reconnection site is continuous. No local acceleration is required to maintain the energetic particle population observed by ISEE1. Thus, contrary to the conclusions in paper 1 , when the bulk of the plasma is considered, there is no justification for excluding the bow shock as a potential source for the 24$44.5 \mathrm{keV}$ ions observed by ISEE-1. Indeed, Figs. 1, 2, and 3 of this comment indicate that the bow shock is a strong candidate for the source of these ions.
Acknowledgements. ISEE-1 Electron data used in the comment are from the CDAWeb and the authors acknowledge the principal investigator for this experiment, K. Ogilvie, for providing these data to the community. Ion composition data were provided by O. W. Lennartsson and are also publically available at www.spasci. com/ISEE_ions/. ISEE-1 and -2 magnetic field data are available at http://www-ssc.igpp.ucla.edu/forms/isee/ and ISEE-3 solar wind data are available at http://www-ssc.igpp.ucla.edu/forms/isee/ 3_form_html. Research at Lockheed Martin was conducted under contracts NNG05GE93G, NNX08AF35G and supported by the National Science Foundation under grant No. 0503201.

Topical Editor I. A. Daglis thanks two anonymous referees for their help in evaluating this paper.

\section{References}

Fuselier, S. A., Berchem, J., Trattner, K. J., and Friedel, R.: Tracing ions in the cusp/LLBL using multi-spacecraft observations and a global MHD simulation, J. Geophys. Res., 107(A7), 1226, doi:10.1029/2001JA000130, 2002.

Ogilvie, K. W., Scudder, J. D., and Doong, H.: The electron spectrometer experiment on ISEE-1, IEEE Trans. Geosci. Electron, GE-16, 261, 1978.

Phillips, J. L., Bame, S. J., Elphic, R. C., Gosling, J. T., Thomsen, M. F., and Onsager, T. G.: Well-resolved observations by ISEE 2 of ion dispersion in the magnetospheric cusp, J. Geophys. Res., 98, 13429-13440, 1993.

Shelley, E. G., Sharp, R. D., Johnson, R. G., et al.: Plasma composition experiment on ISEE-A, IEEE Trans. Geosci. Electron, GE-16, 266, 1978.

Trattner, K. J., Petrinec, S. M., and Fuselier, S. A.: Cusp energetic ions as tracers for particle transport into the magnetosphere, J. Geophys. Res., submitted, 2009.

Whitaker, K. E., Chen, J., and Fritz, T. A.: CEP populations observed by ISEE 1, Geophys. Res. Lett., 33, L23105, doi:10.1029/2006GL027731, 2006.

Whitaker, K. E., Fritz, T. A., Chen, J., and Klida, M.: Energetic particle sounding of the magnetospheric cusp with ISEE-1, Ann. Geophys., 25, 1175-1182, 2007, http://www.ann-geophys.net/25/1175/2007/. 